\title{
ANALYSIS OF DEFORMATION-INDUCED FATIGUE OF THIN-WALLED PLATE GIRDER IN SHEAR
}

\author{
By Ichiro OKURA* and Yukio MAEDA**
}

\begin{abstract}
When a thin-walled plate girder is subjected to repeated shear, it is possible that fatigue cracks may be initiated along the web boundaries in regions where the tension field is anchored. They are caused by the secondary bending stress which is produced by out-of-plane deformation of the web. In this paper, first, the out-of-plane deformation and the secondary bending stress of a rectangular plate under in-plane shear are examined by the finite element analysis. Secondly, using the relation between in-plane shear stress and secondary bending stress, the fatigue strength is expressed in terms of the in-plane shear stress as a function of the web slenderness ratio. And the influence of various factors on the fatigue strength is discussed.
\end{abstract}

\section{INTRODUCTION}

When a thin-walled plate girder is subjected to repeated loading, it is possible that fatigue cracks may be initiated at the toe on the web side of fillet welds connecting the flanges or the vertical stiffeners to the web $^{1}$. The locations of the cracks depend on loading conditions of bending, shear or combined bending and shear. As shown in Fig. 1, when a girder is loaded in shear, the cracks are generally initiated along the web boundaries in regions where the tension field is anchored. They are caused by the secondary bending stress which is produced by out-of-plane deformation of the web under repeated in-plane shear. After the cracks have continued to propagate along the toe of the fillet weld, they branch out into the web in the direction approximately perpendicular to the tension field. The girder will lose its load-carrying capacity due to a reduction of the tension field action, if the cracks are not repaired.

In the past, the authors ${ }^{2)}{ }^{4)}$ carried out theoretical studies on fatigue cracks of thin-walled plate girders in bending. Regarding a web of a girder in bending as a rectangular plate under in-plane bending, and examining its finite out-of-plane deformation by the finite element analysis, they clarified the relation between the initial deflection of the web and the initiation of the $\operatorname{cracks}^{2) .3)}$.

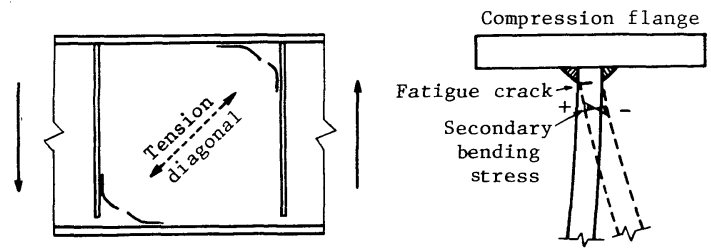

Fig. 1 Fatigue crack locations of thin-walled plate girder in shear.

* Member of JSCE, Dr. Eng., Research Associate, Department of Civil Engineering, Osaka University (Yamada-Oka 2-1, Suita, Osaka 565)

** Member of JSCE, Dr. Eng., Professor, Department of Civil Engineering, Kinki University (Kowakae 3-4-1, Higashiosaka, Osaka 577) 
Furthermore, formulating the relation between in-plane bending stress and secondary bending stress, they expressed the fatigue strength in terms of the in-plane bending stress ${ }^{4)}$.

In this paper, the fatigue due to out-of-plane deformation of the web of a thin-walled plate girder in shear is analyzed. First, the out-of-plane deformation and the secondary bending stress of a rectangular plate under in-plane shear are examined by the finite element analysis. Secondly, using the relation between in-plane shear stress and secondary bending stress, the fatigue strength is expressed in terms of the in-plane shear stress as a function of the web slenderness ratio. And the influence of various factors on the fatigue strength is discussed.

\section{FINITE OUT-OF-PLANE DEFORMATION AND SECONDARY BENDING STRESS OF RECTANGULAR PLATE UNDER IN-PLANE SHEAR ${ }^{5,6)}$}

\section{(1) Analytical Model}

The out-of-plane deflection analysis of a rectangular plate under in-plane shear is carried out by the finite element method $^{2) .31}$. As shown in Fig. 2, the rectangular plate of $a \times b$ is divided into 144 elements. It has an initial deflection in the following form :

$w_{0}=w_{0 \max } \sin (m \pi x / a) \sin (n \pi y / b)$,

where $w_{0}$ is the initial deflection, $w_{0 \max }$ the maximum initial deflection, and $m$ and $n$ the number of half-waves in the $x$ - and $y$-directions, respectively. In this paper, the shape defined by Eq. ( 1 ) for the initial deflection is called the initial

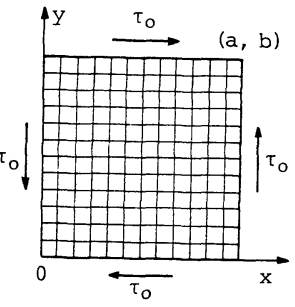

Fig. 2 Mesh division. deflection mode $(m, n)$.

For boundary conditions in the out-of-plane direction along the boundary edges, the following three cases are treated:

(I) All the edges are simply supported.

(II) The two edges of $x=0$ and $a$ are simply supported, and the other two edges of $y=0$ and $b$ are fixed

(III) All the edges are fixed.

For loading conditions, the following two cases are treated :

(a) Forced displacements are applied as follows : $v=-d$ along $x=0, v=d$ along $x=a, u=-d$ along $y=0$, and $u=d$ along $y=b$, where $u$ and $v$ are the displacement components in the $x$ - and $y$-directions, respectively, and $d$ is the forced displacement. In this case, the membrane shear stress at each boundary edge does not show a uniform distribution. Then, the mean shear stress at each boundary edge is given as follows:

$$
\left.\begin{array}{rl}
\tau_{0} & =\frac{1}{a} \int_{0}^{a} \tau_{m} d x \text { at } y=0 \text { and } b \\
& =\frac{1}{b} \int_{0}^{b} \tau_{m} d y \text { at } x=0 \text { and } a
\end{array}\right\},
$$

where $\tau_{0}$ is the mean shear stress, and $\tau_{m}$ the membrane shear stress.

(b) Nodal forces are applied so that the membrane shear stress at each boundary edge can keep a uniform distribution. In this case, all the edges are free to move in the in-plane direction. In the numerical calculation by the finite element method, however, the displacement components $u$ and $v$ at the point $(0,0)$ and $v$ at the point $(a, 0)$, are fixed.

\section{( 2 ) Out-of-Plane Deflection}

Fig. 3 shows the shapes of out-of-plane deflection of a square plate for two different initial deflection modes. The boundary and loading conditions are (I) and (a), respectively. In Fig. 3, $\sigma_{e}$ means the fundamental stress of a plate which is defined as follows: 


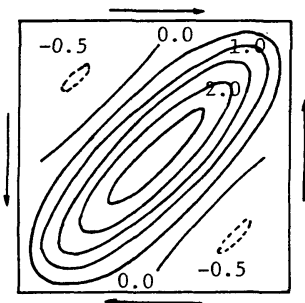

$\tau_{o} / \sigma_{e}=26.58$

$a / b=1.0$

Initial deflection

$\operatorname{mode}(1,1)$

$\mathrm{w}_{\text {omax }} / \mathrm{t}_{\mathrm{w}}=0.1$

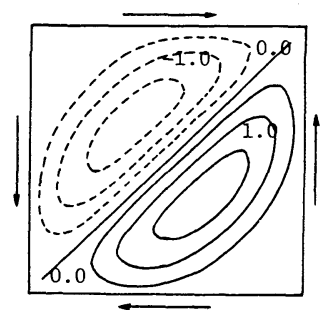

$\tau_{\mathrm{o}} / \sigma_{\mathrm{e}}=27.65$

$\mathrm{a} / \mathrm{b}=1.0$

Initial deflection mode $(1,2)$

$\mathrm{w}_{\text {omax }} / \mathrm{t}_{\mathrm{w}}=0.1$

Fig. 3 Deflection shapes [(I) and (a)].

$$
\sigma_{e}=\frac{\pi^{2} E}{12\left(1-\nu^{2}\right) \beta^{2}},
$$

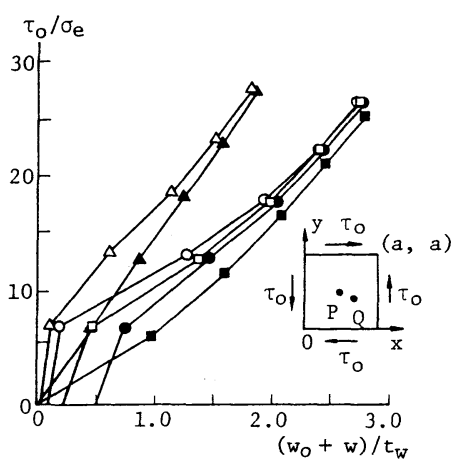

\begin{tabular}{|c|c|c|c|}
\hline \multirow{2}{*}{$\begin{array}{c}\text { Initial deflection } \\
\text { mode }\end{array}$} & \multicolumn{2}{|c|}{$\mathrm{w}_{\text {omax }} / t_{\mathrm{w}}$} & \multirow{2}{*}{ Point } \\
\cline { 2 - 4 } & 0.1 & 0.5 & \\
\hline$(1,1)$ & 0 & $\bullet$ & $\mathrm{P}(\mathrm{a} / 2, \mathrm{a} / 2)$ \\
\hline$(2,2)$ & 0 & $\bullet$ & $\mathrm{Q}(2 \mathrm{a} / 3,5 \mathrm{a} / 12)$ \\
\hline$(1,2)$ & $\Delta$ & $\Delta$ & $\mathrm{Q}$ \\
\hline
\end{tabular}

Fig. 4 Curves of load versus out-of-plane deflection [( I ) and $\left.\left(a^{a}\right)\right]$

where $E$ is Young's modulus, $\nu$ Poisson's ratio, $\beta\left(=b / t_{w}\right)$ the web slenderness ratio, and $t_{w}$ the thickness of the web plate. On the other hand, solid contour lines express the out-of-plane deflection on this side of the plate, while dotted ones express the out-of-plane deflection on the other side of the plate. The figures attached on the contour lines are the magnitude of the out-of-plane deflection to the plate thickness. In the case of the initial deflection mode $(1,1)$, one wave occurs in the direction of the diagonal line of the plate, while in the case of the initial deflection mode $(1,2)$, two waves occur.

Fig. 4 shows the curves of load versus out-of-plane deflection for three different initial deflection modes. $w$ in Fig. 4 means the additional out-of-plane deflection. In the case of the initial deflection modes $(1,1)$ and $(1,2)$, the out-of-plane deflections do not increase so much at the low stage of load, but they increase rapidly after the load has exceeded the buckling load $\left(9.34 \sigma_{e}\right)^{7)}$. In the case of the initial deflection mode $(2,2)$, the deflections increase from the bigining. On the other hand, as the load increases, the curves for the initial deflection modes $(1,1)$ and $(2,2)$ approach one another regardless of the maximum initial deflections, and also the shapes of out-of-plane deflection become similar to one another.

In the cases of the boundary conditions (II) and (III) or of the loading condition (b), the same tendencies as mentioned above can also be observed.

\section{(3) Secondary Bending Stress}

Fig. 5 shows the distributions of secondary bending stress at the boundary edges for the initial deflection modes $(1,1)$ and $(1,2)$. The boundary and loading conditions are (III) and (a), respectively. The symbol $\sigma_{b}$ in Fig. 5 means the secondary bending stress. In the case of the initial deflection mode $(1,1)$, the distributions at the boundary edges are all the same. In the case of the initial deflection mode $(1,2)$, the distributions at $x=0$ and $a$ are the same, and the distributions at $y=0$ and $b$ are the same. And, in the latter case, the maximum secondary bending stress at the edge of $x=0$ is larger than that at the edge of $y=$ $b$. The maximum secondary bending stresses are produced near the corners in the direction of the tension field in both of the initial deflection modes. By the way, Yen ${ }^{8) .9)}$ estimated the secondary bending stress at the toe on the web side of fillet welds around a web by the measured values of the out-of-plane deflection of the web. He showed that the maximum secondary bending stresses occurred near the corners in the direction of the tension field, and that the fatigue cracks were initiated at the locations of the maximum secondary bending stresses. The distributions in Fig. 5 are similar to those estimated by him. 

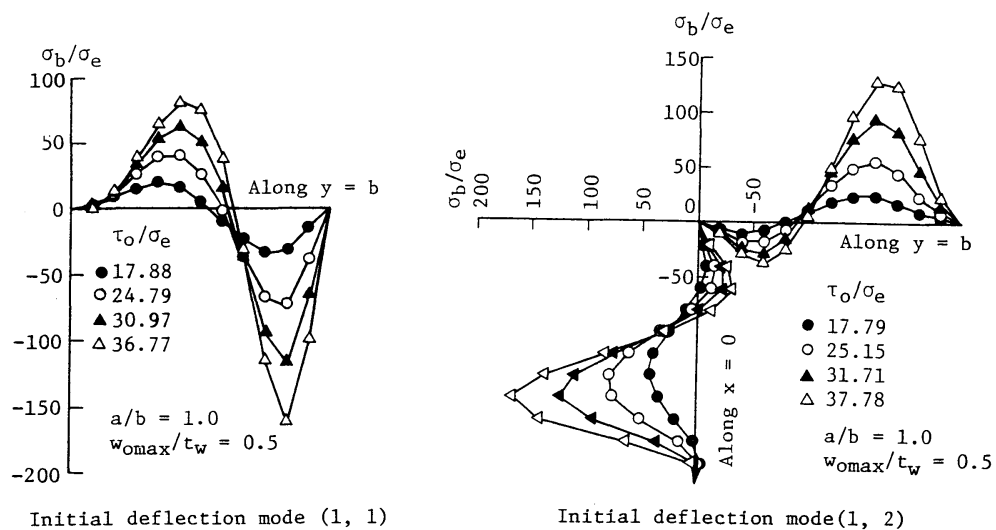

Fig. 5 Distributions of secondary bending stress for $a / b=1.0[($ III $)$ and (a)].

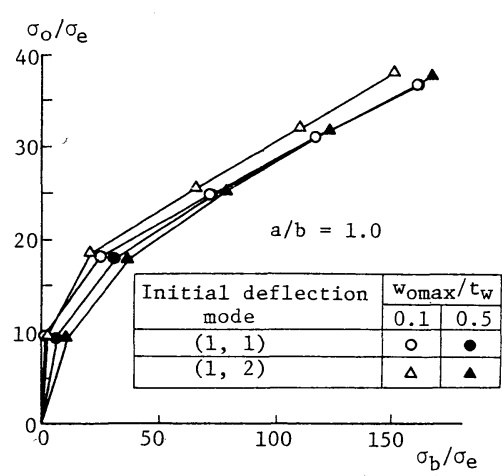

Fig. 6 Curves of load versus maximum secondary bending stress for $a / b=1.0[($ III) and (a) $]$.

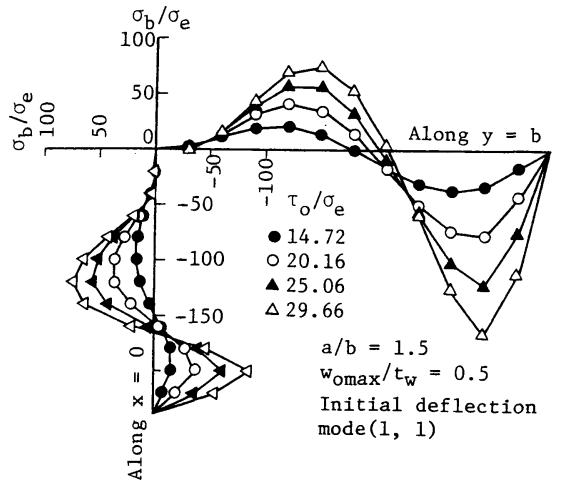

Fig. 7 Distribution of secondary bending stress for $a / b$ $=1.5[(\mathrm{III})$ and $(\mathrm{a})]$.

Table 1 Classification of fatigue crack locations.

\begin{tabular}{c|c|c|c|c|c}
\hline \multirow{2}{*}{ Test } & \multirow{3}{*}{$B$} & \multirow{3}{*}{ a/b } & \multicolumn{3}{|c}{ Fatigue crack locations } \\
\cline { 4 - 6 } & & & $\begin{array}{l}\text { Along } \\
\text { stiffeners }\end{array}$ & $\begin{array}{l}\text { A1 ong } \\
\text { flanges }\end{array}$ & $\begin{array}{l}\text { A1 ong stiffeners } \\
\text { and flanges }\end{array}$ \\
\hline Stal1meyer & 267 & 0.75 & 8 & 1 & 0 \\
\hline \multirow{3}{*}{ Yen } & 246 & 0.5 & 0 & 0 & 0 \\
& to & 1.0 & 10 & 2 & 3 \\
& 287 & 1.5 & 0 & 1 & 1 \\
\hline
\end{tabular}

Fig. 6 shows the relation between load and maximum secondary bending stress. In the case of the initial deflection mode $(1,2)$, the maximum secondary bending stress at the edge of $x=0$ is shown in Fig. 6 . In the case of the initial deflection mode $(1,1)$, the curves for $w_{0 \max } / t_{w}=0.1$ and 0.5 become close to each other as the load increases.

Fig. 7 shows the distribution of secondary bending stress of a rectangular plate of the aspect ratio $a / b=$ 1.5. The initial deflection mode is $(1,1)$. The maximum secondary bending stress at the longer edge is larger than that at the shorter edge. Fatigue tests of thin-walled plate girders in shear were mainly carried out by Stallmeyer ${ }^{10,11)}$ and $\mathrm{Yen}^{8) .9)}$. Table 1 shows a classification of the locations of fatigue cracks observed in their fatigue tests. The figures in the table represent the number of the web panels. When the aspect ratio $a / b$ is smaller than 1.0 , the fatigue cracks are apt to be initiated along the vertical stiffeners, while when it is larger than 1.0 , they are apt to be initiated along the flange. These tendencies can be explained by the aforementioned analytical results.

(4) Influence of In-Plane Bending Stress

Actual plate girders which are subjected to shear are necessarily accompanied by bending. In the fatigue 
tests by $\mathrm{Yen}^{8)}$, he defined the web panels with $\sigma_{0} / \tau_{0}$ less than about 1 as shear panels, where $\tau_{0}$ is the mean shear stress on the web and $\sigma_{0}$ is the bending stress on the flange. Then, the finite out-of-plane deformation of a square plate under combined in-plane shear and bending is examined in the next place.

The boundary condition in the out-of-plane direction is (III), and the in-plane shear is introduced by the loading condition ( $b$ ). The in-plane bending is applied by nodal forces so that the in-plane bending stresses at $x=0$ and $a$ can vary linearly. The calculation is done for $\sigma_{0} / \tau_{0}=1$.

Fig. 8 shows the shapes of out-of-plane deflection for the initial deflection modes $(1,1)$ and $(2,1)$. The shapes resemble each other regardless of the initial deflection modes. A large wave occurs on the diagonal line, and a small one occurs at the upper part on the left. The shapes of out-of-plane deflection for the initial deflection modes $(1,2)$ and $(2,2)$ are also similar to ones in Fig. 8.

The distributions of secondary bending stress resemble one another regardless of the initial deflection modes, because the secondary bending stress which occurs at the boundary edges depends on the shape of out-of-plane deflection. As an example, Fig. 9 shows the distribution of the secondary bending stress for the initial deflection mode $(1,1)$. Fig. 10 shows the relation between load and maximum secondary bending stress at each boundary edge. As the load increases, the difference in magnitude of the maximum secondary bending stresses becomes clearer. Regardless of the initial deflection modes, the maximum secondary bending stress at the edge $y=a$ in compression due to in-plane bending is the largest, and the one at the edge $y=0$ in tension is the smallest.

Fig. 11 shows the relation between load and maximum secondary bending stress at the edge $y=a$ in compression due to in-plane bending for each initial deflection mode. When the load is small, increases in secondary bending stress are different depending on the initial deflection modes. As the load increases,
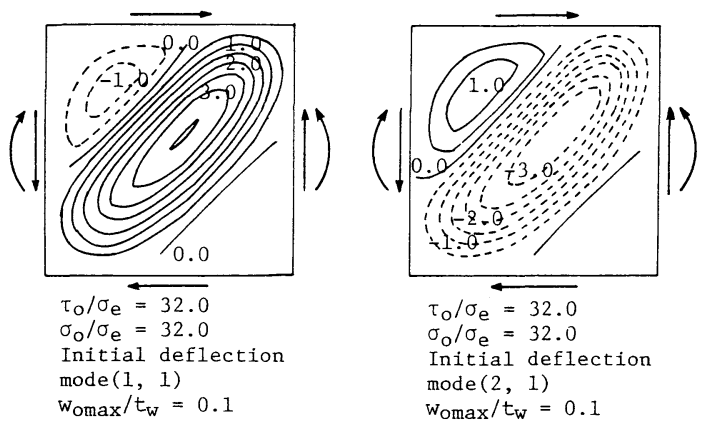

Fig. 8 Deflection shapes in combined in-plane shear and bending.
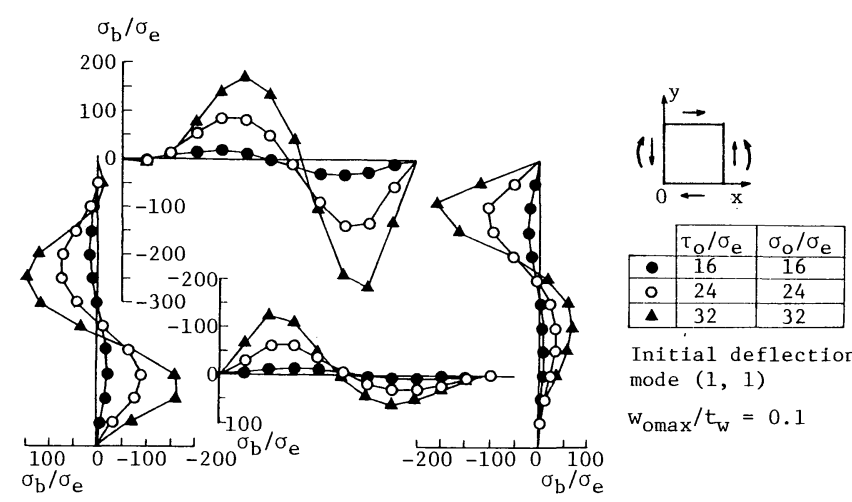

Initial deflection mode $(1,1)$ $\mathrm{w}_{\text {omax }} / t_{\mathrm{w}}=0.1$

Fig. 9 Distribution of secondary bending stress in combined in-plane shear and bending.

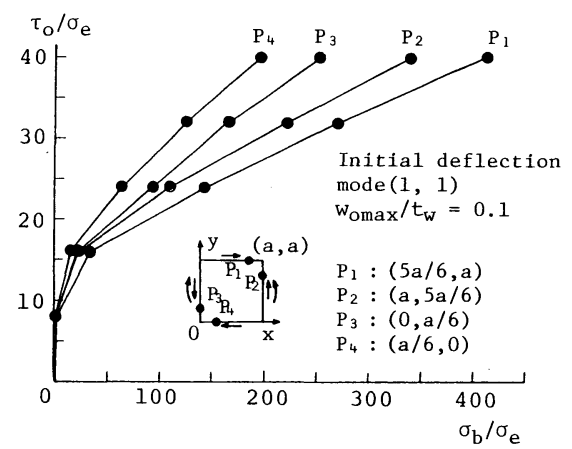

Fig. 10 Curves of load versus maximum secondary bending stress in combined in-plane shear and bending.

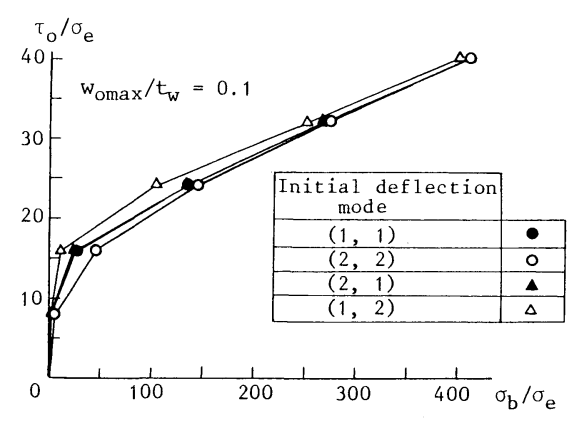

Fig. 11 Curves of load versus maximum secondary bending stress for various initial deflection modes. 
they become close to one another, because the shapes of the out-of-plane deflection are similar to one another regardless of the initial deflection modes.

\section{FATIGUE STRENGTH CONSIDERING OUT-OF-PLANE DEFORMATION OF WEB}

\section{(1) Relation between Load and Secondary Bending Stress of Rectangular Plate under In-Plane Shear}

When a rectangular plate under in-plane shear has the initial deflection mode $(1,1)$, the relation between load and out-of-plane deflection and the relation between secondary bending stress and out-of-plane deflection are respectively given as follows ${ }^{12)}$ :

$$
\begin{aligned}
& \frac{\tau_{0}}{\sigma_{e}}=k_{c r} \eta_{0}+\frac{3}{4}\left(1-\nu^{2}\right) \theta \xi_{0}, \cdots \\
& \frac{\sigma_{b}}{\sigma_{e}}=S \eta_{b}+\sqrt{A^{2} \xi_{0}^{2}+B^{2}}-B,
\end{aligned}
$$

where

$$
\begin{aligned}
& \eta_{0}=\sqrt{\frac{e}{e+r e_{0}}}, \quad \xi_{0}=\left[0.61\left\{\left(\frac{e}{t_{w}}\right)^{2}+2 \frac{e_{0}}{t_{w}} \frac{e}{t_{w}}\right\}^{0.5}+0.39 \frac{e_{0}+e}{t_{w}}\right]\left\{\left(\frac{e}{t_{w}}\right)^{2}+2 \frac{e_{0}}{t_{w}} \frac{e}{t_{w}}\right\}^{0.25} \sqrt{\frac{e+e_{0}}{t_{w}}}, \\
& \eta_{b}=\frac{1}{2}\left\{\frac{e}{t_{w}}+\sqrt{\frac{e}{t_{w}}\left(\frac{e}{t_{w}}+r \frac{e_{0}}{t_{w}}\right)}\right\},
\end{aligned}
$$

$e_{0}=$ the initial deflection at the center of the plate, $e=$ the additional out-of-plane deflection at the center of the plate, $k_{c r}=$ the buckling coefficient, $S=$ an increase in secondary bending stress per an out-of-plane deflection unit of the buckled shape, $r=$ the coefficient depending on the boundary conditions in the out-of-plane direction, $\theta, A, B=$ the coefficients determined by the results of the finite element analysis. The values of $r, k_{c r}, S, \theta, A$ and $B$ are listed in Table 2 for combinations of the boundary conditions ( I ), (II) and (III) and the loading conditions (a) and (b).

\section{( 2 ) Calculation Method of Fatigue Strength}

Using Eqs. (4) and (5), the fatigue strength for the fatigue cracks due to out-of-plane deformation of the web of a plate girder in shear is expressed in terms of the in-plane shear stress. $2 \times 10^{6}$ cycles fatigue strength of fillet welds subjected to secondary bending stress is about $166.7 \mathrm{MPa}$ in stress range ${ }^{13)}$. Letting $\sigma_{b \min }$ and $\sigma_{b \max }$ denote the secondary bending stresses corresponding to the minimum and maximum in-plane shear stresses, $\tau_{0 \min }$ and $\tau_{0 \max }$, respectively, and solving Eqs. (4) and (5) under the condition of

$\sigma_{b \max }-\sigma_{b \min }=166.7$,

the relation between the maximum in-plane shear stress $\tau_{\text {max }}$ for $2 \times 10^{6}$ cycles fatigue strength and the web slenderness ratio $\beta$, can be obtained for the values of the parameter $R$ defined as follows:

$R=\tau_{0 \min } / \tau_{\text {max }}$.

\section{(3) Characteristic of Fatigue Strength}

\begin{tabular}{|c|c|c|c|c|c|c|c|c|c|c|c|c|c|}
\hline & & \multicolumn{4}{|c|}{ (I) } & \multicolumn{4}{|c|}{ (III) } & \multicolumn{4}{|c|}{ (III) } \\
\hline & $a / b$ & 0.5 & 0.75 & 1.0 & 1.5 & 0.5 & 0.75 & 1.0 & 1.5 & 0.5 & 0.75 & 1.0 & 1.5 \\
\hline & $r$ & 1.0 & 1.0 & 1.0 & 1.0 & 0.74 & 0.74 & 0.74 & 0.74 & 0.54 & 0.54 & 0.54 & 0.54 \\
\hline & $\mathrm{k}_{\mathrm{cr}}$ & 26.03 & 13.29 & 9.33 & 7.07 & 26.88 & 15.47 & 12.58 & 11.13 & 42.46 & 21.31 & 14.67 & 11.49 \\
\hline & $\mathrm{S}$ & & & & & 17.51 & 25.60 & 25.27 & 23.79 & 98.46 & 42.90 & 24.32 & 23.82 \\
\hline \multirow{3}{*}{ (a) } & $\theta$ & 5.12 & 4.35 & 3.46 & 2.03 & 5.59 & 4.61 & 3.66 & 2.40 & 8.07 & 5.08 & 3.97 & 2.47 \\
\hline & A & & & & & 15.18 & 15.93 & 14.51 & 10.96 & 19.99 & 25.82 & 17.93 & 12.81 \\
\hline & B & & & & & 40.46 & 36.01 & 33.61 & 36.44 & 0.0 & 111.81 & 71.07 & 62.20 \\
\hline \multirow{3}{*}{ (b) } & $\theta$ & 2.23 & 1.74 & 1.39 & 0.80 & 2.39 & 2.01 & 1.64 & 1.07 & 3.75 & 2.50 & 1.98 & 1.18 \\
\hline & A & & & & & 11.28 & 12.83 & 11.70 & 7.09 & 18.56 & 18.23 & 15.19 & 8.19 \\
\hline & $\mathrm{B}$ & & & & & 76.58 & 60.17 & 53.13 & 27.00 & 0.0 & 111.01 & 115.60 & 47.34 \\
\hline
\end{tabular}

Fig. 12 shows the relation between $\tau_{0 \max } / \tau_{Y}$ and $\beta$ at $R=0$, which is calculated by Eqs. (4), (5) and (6) for $a / b=1.0$ in the boundary condition (III) and the loading condition (b) in Table 2. Here, $\tau_{Y}$ is

Table 2 Values of coefficients 


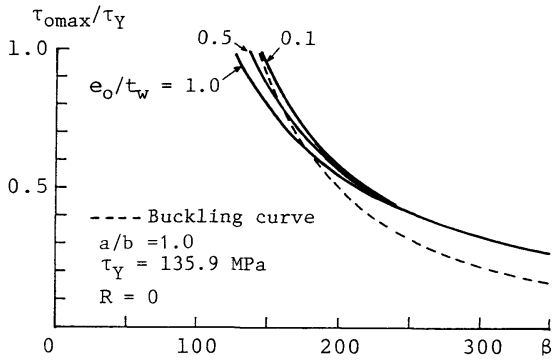

Fig. 12 Curves of $\tau_{0 \max } / \tau_{Y}$ versus $\beta$.

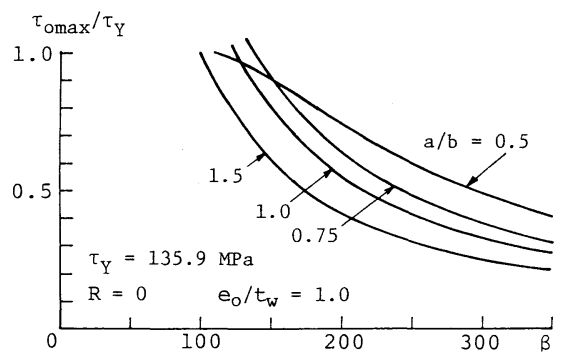

Fig. 14 Influence of aspect ratio.

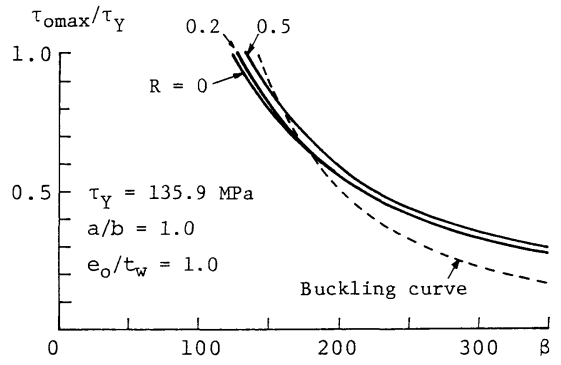

Fig. 13 Influence of stress ratio.

the shear yielding stress. Letting the tensile yielding stress be denoted by $\sigma_{Y}$, and following the yielding condition of von Mises, $\tau_{Y}$ is given by the following equation :

$\tau_{Y}=\sigma_{Y} / \sqrt{3}$.

$\tau_{Y}$ is $135.9 \mathrm{MPa}$ for $\mathrm{SS} 41$ steel of which the specified tensile yielding stress is $235.3 \mathrm{MPa}$. On the other hand, the buckling curves obtained by the following equation is given in Fig. 12:

$\tau_{0 \max }=k_{c r} \sigma_{e}$.

As can be seen in Table 2, the value of the buckling coefficient $k_{c r}$ is 14.67. The followings are pointed out from Fig. 12. The fatigue strength decreases with an increase in the initial deflection in the region of $\beta$ less than about 200. However, when $\beta$ exceeds about 200, it becomes not influenced by the magnitude of the initial deflection, and larger than the buckling strength.

Fig. 13 shows the relations between $\tau_{0 \max } / \tau_{Y}$ and $\beta$ for some values of $R$. The magnitudes of $e_{0} / t_{w}$ are all 1. 0 . It can be seen that the fatigue strengths at $R=0$ and 0.2 approach each other, and that the fatigue strength at $R=0.5$ is slightly larger than those.

Fig. 14 shows the relations between $\tau_{0 \max } / \tau_{Y}$ and $\beta$ for $a / b=0.5,0.75,1.0$ and 1.5. The values of $R$ are all zero. Although the fatigue strength for $a / b=0.5$ is slightly smaller than those for $a / b=0.75$ and 1.0 in the region of $\beta$ from 110 to 150 , the fatigue strength generally increases, as the aspect ratio becomes smaller.

\section{CONCLUSIONS}

In this paper, the fatigue due to out-of-plane deformation of the web of a thin-walled plate girder in shear was analyzed. The main results are as follows:

(1) The shapes of out-of-plane deflection of a rectangular plate subjected to only in-plane shear are different depending on the initial deflection modes. Applying in-plane bending in addition to in-plane shear, however, the shapes of out-of-plane deflection become similar to one another regardless of the initial deflection modes. Accordingly, in the case of the latter, as the load increases, the maximum secondary bending stresses at the boundary edges become not influenced by the initial deflection modes. Actual plate girders in shear are necessarily accompanied by bending. Therefore, it can be concluded that in the plate girders in shear, the influence of the shape of initial deflection on the increase in secondary bending stress, namely, on the initiation of fatigue cracks, is small.

(2) By using the relation between load and secondary bending stress, $2 \times 10^{6}$ cycles fatigue strength for the fatigue cracks due to out-of-plane deformation of the web was expressed in terms of the in-plane shear stress as a function of the web slenderness ratio. This fatigue strength has the following features :

(a) In the region of the web slenderness ratio less than about 200, the fatigue strength decreases with 
an increase in the initial deflection. When the web slenderness ratio exceeds about 200, however, the fatigue strength becomes not influenced by the magnitude of the initial deflection, and larger than the buckling strength.

(b) The fatigue strength becomes larger as the aspect ratio decreases.

(c) The influence of the stress ratio on the fatigue strength is small.

\section{ACKNOWLEDGEMENT}

The authors would like to thank Mr. M. Yoshii (Kobe city) and Mr. H. Hirano (Graduate student of Osaka Univ.) for their help in calculations.

\section{REFERENCES}

1) Maeda, Y. : Fatigue of plate girders, JSSC, Vol.9, No. 86, pp. 32 41, 1973.

2) Maeda, Y. and Okura, I. : Influence of initial deflection of plate girder web on fatigue crack initiation, Proc. of JSCE, No. 319 , pp. 1 11, 1982.

3) Maeda, Y. and Okura, I. : Influence of initial deflection of plate girder webs on fatigue crack initiation, Engineering Structures, Vol. 5 , pp. $58 \sim 66,1983$.

4) Maeda, Y. and Okura, I. : Fatigue strength of plate girder in bending considering out-of-plane deformation of web, Proc. of JSCE, Struct. Eng. /Earthq. Eng., Vol.1, No.2, Japan Society of Civil Engineers, pp. 149 s 159 s, Oct., 1984.

5) Maeda, Y., Okura, I. and Yoshii, M. : Finite out-of-plane behavior of rectangular plates in shear, Technol. Repts. Osaka Univ., Vol.32, No. 1647, pp. 143 150, 1982.

6) Maeda, Y. and Okura, I. : Large deflection analysis of rectangular plates in shear, JSSC, 17th Symposium on Matrix Analysis Methods in Japan, pp. 121 126 and p. 433, 1983.

7) Timoshenko, S. P. and Gere, J.M. : Theory of Elastic Stability, 2nd Edition, McGraw-Hill, pp. 379 385, 1961.

8) Yen, B. T. and Mueller, J. A. : Fatigue tests of large-sized welded plate girders, WRC, Bull. No. 118, Nov., 1966.

9) Mueller, J.A. and Yen, B. T. : Girder web boundary stresses and fatigue, WRC, Bull. No. 127, Jan., 1968.

10) Hall, L. R. and Stallmeyer, J. E. : Thin web girder fatigue behavior as influenced by boundary rigidity, Univ. of Illinois, C. E. Studies SRS No.278, Jan., 1964.

11) Goodpasture, D. W. and Stallmeyer, J. E. : Fatigue behavior of welded thin web girders as influenced by web distortion and boundary rigidity, Univ. of Illinois, C.E. Studies SRS No. 328, Aug., 1967.

12) Maeda, Y., Okura, I. and Hirano, H. : Formulation of finite out-of-plane deformation of rectangular plate in shear, Technol. Repts. Osaka Univ., Vol. 35, No. 1793, pp. 91 100, 1985.

13) Maeda, Y. : Fatigue cracks of deep thin-walled plate girders, Proc. of Bridge Engineering Conference of TRB, USA, Vol.1, pp. 120 128, Sept., 1978.

(Received April 2 1985) 